\title{
Trombosis portal y mesentérica asociada al déficit de la proteína $\mathrm{S}$
}

\author{
J. A. Chirinos Vega, R. Muñoz Gómez, M. Amo Peláez, C. Ibarrola de Andrés y y J. A. Solís Herruzo \\ Departamento de Medicina de Aparato Digestivo. ${ }^{~}$ Departamento de Anatomía Patológica. Hospital Universitario 12 de \\ Octubre. Madrid
}

\section{RESUMEN}

Introducción: la causa más frecuente de trombosis portal (TP) es la cirrosis hepática, mientras que los estados hipercoagulables son raramente identificados como etiología de la TP. Presentamos un caso de TP y mesentérica secundaria al déficit de la proteína $\mathrm{S}$ (pS).

Caso clínico: paciente mujer de 74 años, que debuta con dolor abdominal difuso de 2 semanas de evolución y hemorragia digestiva alta tipo melena secundaria a varices esofágicas. En el estudio se objetiva un hígado heterogéneo, esplenomegalia y ascitis, así como una trombosis portal completa no oclusiva del hilio hepático y de sus ramas y de la vena mesentérica superior con circulación colateral. El estudio etiológico de hepatopatía fue negativo, incluyendo una biopsia hepática que mostraba cambios arquitecturales secundarios al flujo hemático disminuido compatible con hipertensión portal no cirrótica. El estudio de hipercoagulabilidad fue positivo para un déficit de proteína S. pS libre 56\%, pS total $107 \%$. Desde entonces se inició tratamiento anticoagulante sin presentar descompensaciones posteriores.

Discusión: la trombosis portal suele manifestarse con síntomas inespecíficos, siendo la forma de presentación más frecuente la hemorragia digestiva alta como el caso que nos ocupa. La cirrosis es una de las causas más frecuentes de trombosis portal, sin embargo existe hasta un $65 \%$ de estos pacientes que tienen una enfermedad protrombótica asociada, como es el déficit de proteína S. Nuestro caso remarca la importancia de realizar estudios de factores trombogénicos en pacientes con TP, incluso cuando la etiología se puede atribuir a una cirrosis.

Palabras clave: Deficiencia de proteína S. Cirrosis hepática. Trombosis venosa. Vena porta.

\begin{abstract}
Introduction: liver cirrhosis is the main cause of portal thrombosis (PT), while hypercoagulability syndromes are rarely found as the etiology of PT. We report a case of portal and mesenteric thrombosis secondary to protein S deficiency.

Case report: a 74-year-old woman was admitted with melena secondary to upper gastrointestinal bleeding. She reported mild, diffuse abdominal pain in the last 2 weeks. Endoscopy revealed ruptured esophageal varices. Doppler ultrasonography and CT demonstrated a heterogeneous liver, splenomegaly and ascites, and complete non-occlusive PT involving the hilum and portal branches, as well as the superior mesenteric vein, with portosystemic collaterals. At this point a complete study for cirrhosis etiologies was negative, including a liver biopsy that showed nonspecific architectural changes secondary to diminished blood flow, which suggested noncirrhotic portal hypertension. The search for hypercoagulability states determined a deficiency of $\mathrm{S}$ protein, with total $\mathrm{pS}=107 \%$ and free $\mathrm{pS}=56 \%$. The patient was started on anticoagulant treatment and no other thrombotic events occurred.

Discussion: PT usually manifests without specific symptoms. The most common presentation is upper gastrointestinal bleeding, as occurred in our patient. Liver cirrhosis is one of the most frequent cause of PT. Up to $65 \%$ of these patients present an associated prothrombotic state, including protein $\mathrm{S}$ deficiency. Our case reminds us of the importance of a systematic search for hipercoagulability syndromes in patients with TP, even when the etiology can be conferred to liver cirrhosis.
\end{abstract}

Key words: Protein S deficiency. Liver cirrhosis. Venous thrombosis. Portal vein.

Chirinos Vega JA, Muñoz Gómez R, Amo Peláez M, Ibarrola de Andrés C, Solís Herruzo JA. Trombosis portal y mesentérica asociada al déficit de la proteína S. Rev Esp Enferm Dig 2008; 100: 104-107.

Recibido: 08-11-07.
Aceptado: $15-11-07$

Correspondencia: Juan Antonio Chirinos Vega. Paseo Marqués de Zafra, 12,3ํㅡ. 28028 Madrid. e-mail: juanochirinos@hotmail.com

\section{INTRODUCCIÓN}

La trombosis portal y mesentérica (TPyM) es un evento poco frecuente que está asociado muchas veces a un factor predisponente local, lo que hace fácil su diagnósti- 
co. Sin embargo existen casos asintomáticos, sin causa predisponente claramente establecida, que se quedan muchas veces sin diagnóstico o con el diagnóstico de origen idiopático. Es en estos casos donde los estados de hipercoagulabilidad, tales como el déficit de la proteína $S$ (DPS), se deben descartar. Existen pocos casos publicados como el descrito por nosotros, no sólo por su baja incidencia sino también por la falta de una aproximación diagnóstica sistemática para el estudio de este problema, lo que hace suponer un infradiagnóstico de casos.

Presentamos un caso de TPyM con clínica de hemorragia digestiva alta e hipertensión portal como la primera manifestación del DPS.

\section{CASO CLÍNICO}

Paciente mujer de 74 años que ingresó por cuadro de dolor abdominal difuso, hiporexia y astenia de 2 semanas de evolución. Presentaba vómitos en posos de café y heces melénicas desde hacía 2 días. Como antecedentes personales refería: hipertensión en tratamiento con amiloride/hidroclorotiazida y dislipemia que controlaba con lovastatina. No era fumadora y negaba la ingesta de AINE, alcohol o anticonceptivos orales. Sus antecedentes familiares eran: madre fallecida de cancer gástrico, hermano con cirrosis hepática de etiología desconocida y hermana con carcinoma hepatocelular.

- Exploración física: estabilidad hemodinámica. Dolor difuso a la palpación abdominal, sin signos de peritonismo.

-Analítica al ingreso: hemoglobina: $13,5 \mathrm{mg} / \mathrm{dl}$; hematocrito: $37,6 \%$; plaquetas: 120.000 plaquetas/ $\mu$; bilirrubina: $247 \mathrm{mg} / \mathrm{dl}$; fosfatasa alcalina: $220 \mathrm{UI} / 1$; aspartato aminotransferasa: $59 \mathrm{UI} / 1$; alanina aminotransferasa: $28 \mathrm{UI} / 1$ y gamma-glutamil transpeptidasa: $129 \mathrm{UI} / \mathrm{l}$. Se realizó una gastroscopia de urgencia donde se evidenciaron varices esofágicas de mediano tamaño sin signos de alarma de sangrado inminente [F2 RC(-) según la clasificación de la Sociedad Japonesa para el Estudio de Hipertensión Portal] y múltiples erosiones con fibrina a nivel gástrico y duodenal sin signos de hemostasia reciente. En la tomografía computerizada y en la ecografía abdominal el hígado era de tamaño normal, de bordes levemente ondulados con un parénquima discretamente heterogéneo, sin lesiones ocupantes de espacio. A su vez se objetivó esplenomegalia, ascitis y derrame pleural izquierdo de escasa cuantía. Por otro lado, existía una trombosis portal completa no oclusiva a nivel del hilio hepático y de sus ramas, así como otro trombo a nivel de la vena mesentérica superior con presencia de circulación colateral en los ligamentos gastrohepático y periesplénico (Fig. 1). El estudio etiológico de las diferentes causas de hepatopatía crónica incluyó: virus hepatotropos (VHB, VHC) y VIH, estudio de autoinmunidad, ceruloplasmina, ferritina y porfirinas en orina, siendo todos los resultados negativos. Posterior-

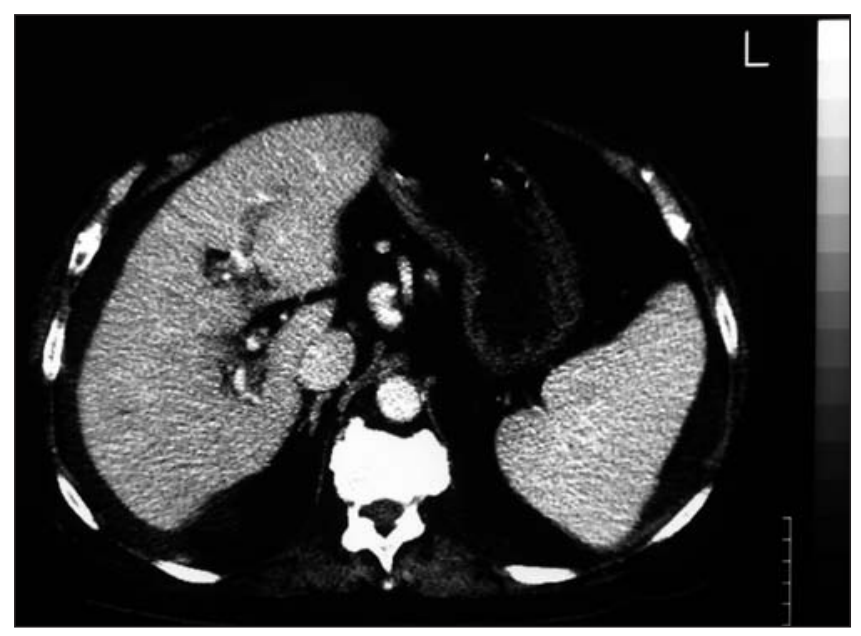

Fig. 1. Tomografia axial computerizada con contraste endovenoso, en la que se pueden observar signos de trombosis de las ramas portales.

mente se realizó una biopsia hepática percutánea con estudio hemodinámico que mostraba un gradiente de presión portal de $12 \mathrm{mmHg}$ con flebografía normal. La biopsia mostró una alteración de la arquitectura hepática debida a hiperplasia hepatocitaria en la zona 1 de algunos de los acinos, con tendencia a ser nodular pero sin septos de fibrosis perinodulares. Los tractos portales mostraban la triada ducto-arteria-vena habituales, aunque la vena era a veces poco patente y se observaba alguna vena paraportal. Presentaban leve ampliación fibrosa y contenían leve infiltrado inflamatorio linfoplasmocitario con desbordamiento focal por la interfase periportal. En el lobulillo se observaba también algún foco inflamatorio, si bien no había cuerpos acidófilos ni otros hallazgos relevantes (Figs. 2 y 3). Estos cambios fibroinflamatorios leves, la hiperplasia nodular irregular así como la ausencia de fibrosis perinodular descartan la presencia de cirrosis y son compatibles con una hipertensión portal no cirrótica. Ante la posibilidad

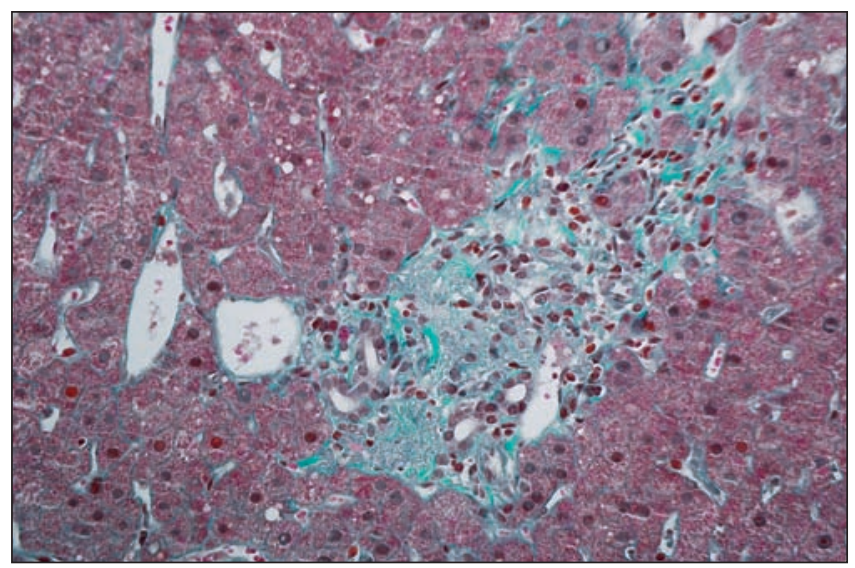

Fig. 2. Masson tricrómico (x400). Tracto porta con ampliación fibrosa, vena porta poco patente y alguna vena paraportal. 


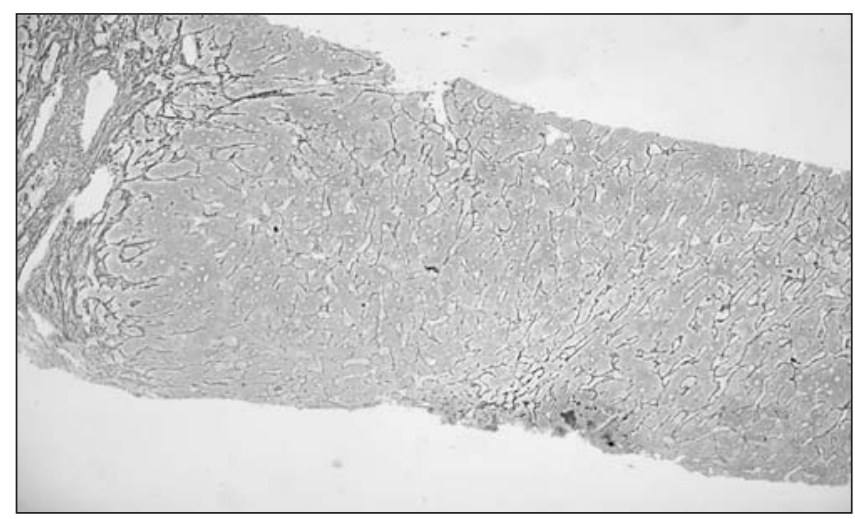

Fig. 3. Plata de Wilder (x200). Reticulina mostrando un foco de hiperplasia hepatocitaria nodular con la trabeculación doble hepatocitaria.

de que todos los cambios hepáticos fuesen secundarios a una trombosis portal primaria se realizó un estudio de hipercoagulabilidad.

Este estudio demostró una proteína $\mathrm{S}(\mathrm{pS})$ libre antigénica, pS total antigénica y pS libre anticoagulante de 56, 107 y $44 \%$ respectivamente, lo cual era sugestivo de un déficit de proteína $\mathrm{S}$ tipo I. El resto de parámetros de la coagulación (que incluyeron fibrinógeno, antitrombina, proteína $\mathrm{C}$, anticuerpos anticardiolipina, resistencia a la proteína $\mathrm{C}$ activada, factor V Leyden, factor II G 20210A y homocisteína) se encontraban en el rango de la normalidad. Este resultado fue confirmado en un control posterior.

A la paciente se le instauró tratamiento anticoagulante con acenocumarol y diuréticos de forma indefinida. En el seguimiento de la paciente no se han evidenciado nuevas descompensaciones.

\section{DISCUSIÓN}

La trombosis del eje esplenoportal (TP) es un evento raro que muchas veces se manifiesta de forma subaguda o crónica con síntomas relacionados con la hipertensión portal. Cuando aparecen los síntomas, estos son inespecíficos e incluyen vómitos, dolor abdominal, aumento del perímetro abdominal, fiebre y hemorragia digestiva alta secundaria a sangrado por varices, siendo esta última presentación la más frecuente $(1,2)$.

La etiología de la TP se puede agrupar en tres categorías: la primera en relación con alteraciones trombofílicas sistémicas adquiridas (síndromes mieloproliferativos primarios, síndrome antifosfolípido, la hemoglobinuria paroxística nocturna y la hiperhomocistinemia) o hereditarias (mutación del factor $\mathrm{V}$ de Leiden, mutación G20210A del gen de la protrombina, déficit de las proteínas $\mathrm{C}$ y $\mathrm{S}$ y la deficiencia de antitrombina) (3-5). En el segundo grupo se incluyen los factores de riesgo de trombosis como son el uso de anticonceptivos orales y el embarazo. El tercer grupo es el relacionado con factores locales: lesiones inflamatorias (onfalitis, diverticulitis, colecistitis, entre otras) y agresiones al eje esplenoportal (esplenectomía, colectomía, etc.). El $60 \%$ de los casos diagnosticados de TP son causados por factores trombogénicos sistémicos y el $40 \%$ por factores locales. La coexistencia de factores etiológicos múltiples sucede en el 15\% de los pacientes y ello obliga a la búsqueda de factores trombogénicos sistémicos, aun habiendo sido reconocida la existencia de factores trombogénicos locales y viceversa $(3,6)$. La cirrosis es la causa más frecuente de TP actualmente. Sin embargo es importante remarcar que hasta un $69,5 \%$ de los pacientes con cirrosis presentan un estado de hipercoagulabilidad hereditario (7).

En el estudio de Egesel y cols. (8) se revisaron 23 pacientes con trombosis portal por hipercoagulabilidad y se encontró que hasta un $43 \%$ de los casos tenía un DPS, similar al caso que nos ocupa.

La proteína $\mathrm{S}$ es sintetizada por los hepatocitos y los megacariocitos y es dependiente de la vitamina K. Actúa como cofactor de la proteína $\mathrm{C}$. El $50 \%$ circula libre y el otro $50 \%$ unido a la proteína $\mathrm{C} 4 \mathrm{~b}$. La deficiencia cuantitativa o cualitativa de proteína $\mathrm{S}$ se transmite de manera autosómica dominante. Un $74 \%$ de los pacientes desarrolla una trombosis venosa profunda a diferentes niveles. La incidencia anual es del $1 \%$ y en el $56 \%$ de los episodios ocurre de manera espontánea. El riesgo relativo de trombosis es de 8,5. La mitad de los pacientes que desarrollan una trombosis lo hacen antes de los 25 años (9).

Existen dos formas descritas de DPS. El tipo I está caracterizado por tener un nivel de proteína $\mathrm{S}$ total normal y un nivel bajo de proteína $\mathrm{S}$ libre. En el tipo II ambos niveles están bajos. Los factores precipitantes de un efecto trombótico pueden ser la inmovilización, la cirugía y los anticonceptivos orales. Sin embargo en la mayoría de las ocasiones no se identifica ninguno de estos factores (10-12).

En esta paciente se descartaron razonablemente las principales causas de deficiencia adquirida de proteína $S$ (síndrome nefrótico, CID, estrógenos orales, síndrome inflamatorio, enfermedad por VIH o disfunción hepatocelular), por lo que el origen más probable del defecto sería el hereditario.

En la biopsia no es frecuente observar los signos característicos de vasculopatía portal obliterativa. Al ser la afectación parcheada, no siempre aparecen los cambios venosos fibroobliterativos y/o la desaparición de la vena porta y rara vez se pueden ver trombos en las venas pequeñas. Más frecuentemente son los hallazgos de hiperplasia y atrofia hepatocitaria los que indican un aporte sanguíneo irregular y orientan al diagnóstico. Los cambios histológicos pueden ser muy sutiles y la presencia de fibroinflamación leve puede confundirse con otras hepatopatías $(13,14)$. Por tanto, el clínico y el patólogo deben pensar en la posibilidad de vasculopatía portal para su correcto diagnóstico. La primera manifestación de la TP no 
es siempre la hipertensión portal como en el caso que hemos presentado. Puede presentarse con una pequeña alteración bioquímica mantenida, generalmente una colestasis disociada y desarrollarse más tarde la hipertensión portal. Por tanto, ante un cuadro de colestasis sin otra etiología que lo explique y con una biopsia sin necroinflamación relevante y sin datos de colangiopatía, esteatohepatitis o fibrosis avanzada, debe considerarse la posibilidad de vasculopatía portal y deben estudiarse los factores trombogénicos como una de las causas más probables de la misma. También debe tenerse en cuenta que un porcentaje de las hipertensiones portales no se deben a cirrosis. La transformación arquitectural en el hígado con vasculopatía portal varía mucho en severidad y extensión. Puede mostrar una imagen de micronodulación y/o macronodulación focales o difusas que puede conducir a un falso diagnóstico clínico de cirrosis y/o incluso de lesión ocupante de espacio. La biopsia es por tanto esencial para no confundir estos cuadros.

En el caso de nuestra paciente, los hallazgos histológicos son probablemente secundarios a la trombosis y no de manera contraria. Sin embargo es importante recordar que muchos de los pacientes con hiperplasia nodular regenerativa requieren más de una biopsia para realizar el diagnóstico histológico y que esta patología no se puede descartar del todo (15). En la cirrosis hepática la TP se asocia a un grado de fibrosis elevado que ocasiona un flujo portal enlentecido y favorece la aparición de la trombosis, hallazgos que nuestra paciente no presentaba.

Nuestro caso corrobora que el déficit de pS es un factor de riesgo para la TPyM y que debe realizarse un estudio de factores trombogénicos sistémicos a pesar de la existencia de factores locales.

\section{BIBLIOGRAFÍA}

1. Wang JT, Zhao HY, Liu YL. Portal vein thrombosis. Hepatobiliary Pancreat Dis Int 2005; 4: 515-8.

2. Sobhonslidsuk A, Reddy KR. Portal vein thrombosis: A concise review. Am J Gastroenterol 2002; 97: 535-41.

3. Sarin SK, Agarwal SR. Extrahepatic portal vein obstruction. Semin Liver Dis 2002; 22: 43-58.

4. Webster GJ, Burroughs AK, Riordan SM. Review article: Portal vein thrombosis-new insights into aetiology and managment. Aliment Pharmacol Ther 2005; 21: 1-9.

5. García-Pagan JC, Hernández-Guerra M. Trombosis Portal. Panel de Expertos Octubre 2004. Disponible en: http://www.hepatonet.com/ formación/expertos19.php

6. Primignani M, Martinelli I, Bucciarelli P, Battaglioli T, Reati R, Fabris F, et al. Risk factors for thrombophilia in extrahepatic portal vein obstruction. Hepatol 2005; 41: 603-8.

7. Amitrano L, Brancaccio V, Guardascione MA, et al. Inherited coagulation disorders in cirrhotic patients with portal vein trombosis. Hepatol 2000; 31: 345-8.

8. Egesel T, Buyukasik Y, Dundar SV, Gurgey A, Kirazli S, Bayraktar $\mathrm{Y}$. The role of natural anticoagulant deficiencies and factor $\mathrm{V}$ Leiden in the development of idiopathic portal vein thrombosis. J Clin Gastroenterol 2000; 30: 66-71.

9. Thomas RH. Hypercoagulability syndromes. Arch Intern Med 2001; 161: 2433-9.

10. Zigrossi P, Campanini M, Bordin G, Arceci F, Gamba G, Gnemmi $\mathrm{PM}$, et al. Portal and mesenteric thrombosis in protein $\mathrm{S}(\mathrm{pS})$ deficiency. Am J Gastroenterol 1996; 91: 163-5.

11. Godeau B, Leroy-Matheron C, Gouault-Heilmann M, Schaeffer A. A case of portal vein thrombosis associated with protein S deficiency. J Hepatol 1993; 18: 258

12. Tomás M, García C, Marcos MS, Pérez Arellano E, Morillas JD, Castellano-Tortajada G. Trombosis de las venas porta y mesentérica superior asociada a déficit de proteína S. Rev Esp Enferm Dig 2000; 92: 177-8.

13. Ibarrola C, Colina F. Clinicopathological features of nine cases of non-cirrhotic portal hypertension: Current definitions and criteria are inadequate. Histopathol 2003; 42: 251-64.

14. Roskams T, et al. Histopahology of portal hypertension: A practical guideline. Histopathol 2003; 42: 2-13.

15. Colina F, Alberti N, Solís JA, Martínez Tello J. Diffuse nodular regenerative hyperplasia of the liver (DNRH). A clinicopathologic study of 24 cases. Liver 1989; 9: 253-65. 